\title{
Haptically assisted chemotaxis for odor source localization
}

\author{
Ricardo Roberts $^{1}$ [D $\cdot$ B. Lorena Villarreal ${ }^{1}$. Ernesto Rodriguez-Leal ${ }^{1} \cdot$ J. L. Gordillo ${ }^{1}$
}

(c) Springer Nature Switzerland AG 2019

\begin{abstract}
Detection of chemical trails has multiple applications in industry and exploration, where prompt localization of dangerous substances encourages development of robust sensors, systems, and algorithms. Current chemical sensing robotics has focused on autonomous navigation, while transduction of chemical information to visual, tactile, or other types of sensory cues have not been thoroughly addressed. This work proposes the inclusion of a human operator in order to solve the robot navigation problem and proposes visual and haptic feedback arrangements that carry the information transmitted by the chemical sensors. A chemical source is placed in a simulated environment, which is navigated by a tracked robot controlled by human drivers. A multipoint haptic interface operates as the robot controller and provides force feedback according to the direction of the chemical gradient detected by the robot. Seven experiments test the tracking performance of three combinations of haptic and visual feedback under different air current configurations, which consistently prove the feasibility of a chemotaxis-based navigation system. Moreover, this work demonstrates with statistical significance that gradient information transmitted using haptic feedback minimizes the required time for reaching the chemical source. This enables the operator's visual capability in other important tasks during navigation (i.e., obstacle avoidance).
\end{abstract}

Keywords Haptics · Olfaction · Human-computer interaction · Teleoperation · Assisted navigation · Simulation

\section{Introduction}

Living organisms depend on chemical detection for their survival. Mammals in particular have highly developed chemoreceptors throughout their bodies. Various examples include the detection of hormones by various organs of the endocrine system [25], or the ability of the carotid body to measure $\mathrm{CO}_{2}$ levels in the blood. The most known chemical receptors are, however, the senses of gustation (taste) and olfaction (smell). Both senses have been the subject of ongoing research due to their complexity.

Similarly, replicating the sensitivity and selectivity of both senses, particularly the sense of smell, opens an enormous potential in a wide set of applications by robotic systems in industrial environments (e.g., detection of chemical leaks and continuous air quality monitoring).
Chemoreceptor-based navigation is used by both unicellular organisms [10] as well as their multicellular counterparts. Specifically, chemotaxis refers to movement of organisms (-taxis) due to a chemical (chemo-). In the Mammalia class, the sense of smell of the Ursidae family (bears) is one of the most developed, being able to navigate toward food that is several miles away [16]. Similarly, mechanoreceptor-based navigation is also widely used by organisms as diverse as bacteria provided with mechanosensitive channels [41] to star-nosed moles, which have highly sensitive snout appendages that use to navigate in light-deprived environments [12]. The above-mentioned mechanoreceptors work alongside propioceptors located throughout muscles that monitor the joints configuration in order to produce awareness of various environmental characteristics during locomotion. Note, however, that

$\bowtie$ Ricardo Roberts, robertsu@mit.edu| ${ }^{1}$ Tecnologico de Monterrey, Escuela de Ingenieria y Ciencias, Monterrey 64849, Mexico.

SN Applied Sciences (2019) 1:382 | https://doi.org/10.1007/s42452-019-0411-x

Received: 6 February 2019 / Accepted: 25 March 2019 / Published online: 30 March 2019 
the concept of chemotaxis can be extrapolated to other agents such as unmanned vehicles [50].

Both tactile and olfactory interactions have been studied separately in navigation-related research. Haptic devices can communicate by applying vibration or static forces to the skin [2] and have been used in assisted navigation $[3,9,58]$. Examples include the search for enhanced usability in teleoperated robots $[37,63]$, the use of shapechanging haptic interfaces to help pedestrians [59] or vibrotactile actuated belts for soldiers [20] and the visually impaired [29]. Training on simulations $[6,26,51,57]$ and controlled environments [15] represent popular applications of haptic technology. In an example of the latter, a joystick provided with force feedback is used to train physically impaired toddlers to navigate through an predefined trajectory [4], an actuator filled seat provides haptic feedback to a car driver [13], and a vibrotactile armband guides blindfolded subjects through crowded environments [8]. Furthermore, other researchers have focused on user interface accessibility $[7,14]$, algorithm design in haptic devices $[5,30,66]$, and their inclusion with other technologies such as virtual reality [21, 24].

Haptics has found a niche application in unmanned aerial vehicles (UAVs) navigation, which has received attention in the last decade [33, 36], particularly quadcopters, which have reduced the costs and dangers involved in their use. Haptic-enhanced UAV navigation research focuses on the implementation of force feedback algorithms to enhance the capability to avoid obstacles [33,53]. These tactationbased navigation systems provide information to a human operator that has the final control over the device; however, this scheme has not been used in olfaction-based arrangements.

Olfaction-based algorithms have been implemented in different robotic platforms [22] and environments [47]; for example, odor sensors are used as instruments in terrestrial autonomous vehicles $[45,46]$, which are programmed with different algorithms in order to reach a given odor source [40]. Chemical sensors have also been installed on aerial vehicles, e.g., Neumann et al. have operated an UAV through a predefined path to map the concentration of $\mathrm{CO}_{2}$ in a determined area around a $\mathrm{CO}_{2}$ source [49]. Note that this application estimates gas concentration in multiple points but uses a single sensor. Although a single reading detects the presence of a chemical, a system with multiple lectures enables the determination of odor gradient, which is indispensable for the source localization [64]. Gradient awareness is possible with the use of multiple sensors placed in distinct parts of the robot, which detect subtle changes in odor concentration $[39,44,60$, 61]. The effectiveness to discern the chemical direction is called directionality or directivity [60], which depends on the robot design $[39,42,61]$. Effective directivity could be used in various applications such as detecting toxic gases in mines or drugs and explosives in airports. Search and rescue operations benefit from this technology by locating individuals trapped in buildings during disaster situations, where time is critical [62]. These scenarios are particularly difficult for robotic exploration, since their environment is not controlled as in a laboratory; features such as changing wind patterns alter the distributions of the chemicals unpredictably.

The particular case of chemical plume tracking is thoroughly studied using autonomous vehicles, although has received less attention from the research community focused on teleoperated systems and human-machine interaction. Proposed methods that detect chemical sources in environments with air currents include singlerobot [11] and multiple-robot (swarm) systems [65]. Note that these approaches rely on algorithms for the robot navigation, which can be overwhelmed by the complexity of certain situations (i.e., a momentaneous wind gust affecting the sensors); in contrast, a human operator can understand these types of changes and act accordingly. Therefore, this work utilizes force feedback to inform a driver about the perceived chemical gradient in the robot surroundings, while the operator is instructed to reach the chemical source by using the haptic information to navigate toward the origin. This approach does not use any type of novel sensor or actuator; however, note that even if olfaction and touch have been studied separately in multiple examples, applications that incorporate both senses have not been properly examined to the best of the authors knowledge.

Teleoperated robots can go to places dangerous or outright unsuitable to humans such as mines, disaster areas, toxic environments, radioactive zones, and outer space. Research has focused on hardware and algorithms to explore said places using without human intervention [48]. Missteps, however, are unavoidable. For example, on April 2015, an autonomous robot got trapped inside of a reactor in the inhospitable area of Fukushima [1], incident similar to another robot lost on October 2011 [31]. These incidents show a need of improvement in robotic maneuvering in such scenarios. On the other hand, a study realized on 2013 [52] mapped all the gas leaks found on the urban pipeline of city of Boston, where high concentrations of natural gas and methane where discovered at landfill sites, wetlands, and sewer systems which are difficult scenarios for humans to work. Instead, a robot can substitute them to fix the leaks.

The purpose of this work is to demonstrate that such capabilities facilitate odor source localization tasks, and it also encourages further research on multiple sensoryassisted navigations, which has received little attention. The main contribution of this work is the analysis of the 
performance reached by a human operator combining different arrangements of smell, vision, and touch capabilities using haptic or visual information as feedback for odor directionality. It is observed that tactile information allows proper navigation; hence, the sense of sight can be used in other tasks such as controlling obstacle avoidance or finding other kind of targets.

The remainder of the article is organized as follows: The document analyzes the odor source localization problem and studies the equations governing fluids and the odor dispersion in such medium. The next section gives an overview of the systems used in this work and their interaction with the user and between each other. The document continues with an analysis of the haptic device and the proposed transduction from olfactory information to force feedback behavior. The following section explains the elements of the simulated navigation experience, which include the chemical dispersion behavior, the robot and sensor simulation, and the visual human-machine interface (HMI). The document then explains the performed experiments and discusses the obtained results. The document ends with conclusions and suggestions for further work.

\section{Odor source localization task}

Unmanned vehicles perceive and navigate their surroundings using various sensors and actuators $[28,43]$. An individual can manipulate such devices remotely, although autonomous operation is also possible with the use of proper algorithms. In either case, these devices set a distance between the operator and the environment, representing a valuable feature in remote or dangerous situations. Land navigation has received particular attention by the research community in the past decades. For instance, self-parking and self-driving cars caught the attention of the automotive industry; unmanned vehicles, however, have found a niche in search and rescue applications. These environments change continuously and unpredictably; therefore, data gathering is critical for the proper control actions to be taken, which have to be quick, accurate, and safe for any human near the vehicle. Particular problems in search and rescue operations comprise leak detection (i.e., volatile or poisonous gases) and victim localization. Both tasks are currently performed by handheld devices and trained dogs, respectively; however, both approaches require the involvement of a human, limiting the range in which such searches can be performed. Conversely, chemical-sensitive robotic devices could be deployed in environments that represent a threat to humans and dogs. The development of such devices need an exhaustive understanding of fluid behavior, particularly chemical dispersion.

Crank [17] describes diffusion as the process by which matter is transported in a medium due to random molecular motion. This mechanism homogenizes the environment by transporting a chemical from high- to low-concentration regions. Diffusion is modeled by Fick's first law (1) for a solute dispersing in a homogeneous medium or by the Maxwell-Stefan diffusion used in systems comprised by various components. This work assumes a homogeneous medium interacting with the measured chemical; therefore, Fick's first law is used. This law describes the diffusion flux $J$, which is the amount of material that passes through a given area in a given amount of time. This model states that the diffusion flux $J$ is proportional to the concentration gradient $\frac{\partial \phi}{\partial x}$. The direction of this flux, however, is opposite to the gradient, and therefore, a minus sign is inserted into the equation. This negatively signed gradient is multiplied by the diffusion coefficient of the medium, denoted by $D$, which is a proportionality constant expressed in distance squared over time. Its exact value can be estimated using the Arrhenius, Stokes-Einstein, or Boltzmann equations for solid, liquid, or gaseous mediums, respectively. In one spatial dimension, denoted as $x$, the law is expressed as:

$J=-D \frac{\partial \phi}{\partial x}$

where $\phi$ is the amount of substance in a given volume (i.e., concentration) and $x$ is the spatial displacement. Additionally, Fick's second law predicts how concentration changes with time $t$ due to diffusion:

$\frac{\partial \phi}{\partial t}=D \frac{\partial^{2} \phi}{\partial x^{2}}$

Furthermore, the general form for the diffusion-advection equation $[17,23,38]$ presents two components: the diffusion flux per unit area per unit time and the advective flux per unit area per unit time. A three-dimensional Cartesian coordinate system is expressed as:

$$
\frac{\partial \phi}{\partial t}=D\left(\frac{\partial^{2} \phi}{\partial x^{2}}+\frac{\partial^{2} \phi}{\partial y^{2}}+\frac{\partial^{2} \phi}{\partial z^{2}}\right)-\left(u_{x} \frac{\partial \phi}{\partial x}+u_{y} \frac{\partial \phi}{\partial y}+u_{z} \frac{\partial \phi}{\partial z}\right)
$$

where $u_{x}, u_{y}$, and $u_{z}$ are the velocity components of the diffusive medium in their three corresponding axes of spatial displacement, $x, y$, and $z$, respectively.

Smell sensors implemented in mobile robots started in 1984 with the use of chemical-sensitive robots in the nuclear industry [34]. There are many algorithms used to support and increase the efficiency of odor source localization. These are most commonly classified by the terms of chemotaxis and anemotaxis depending on the 
environment and the capabilities of the odor sensors. Chemotaxis, as stated in Sect. 1, refers to the movement triggered by chemicals in the environment of a organism. Similarly, anemotaxis refers to the detection of flow and its direction as navigational compass [27, 32]. Both strategies react to different environmental features, but assume favorable conditions such as the existence of continuous chemical flow and the absence of local maxima of the chemical of interest. Assumptions also made by the work presented in this document. The resulting environment is used to explore various forms of assisted navigation, particularly the transduction of chemical information to visual and tactile cues. Thus, assisted navigation represents an important contribution for odor source localization since a human operator can detect variations of the medium and act accordingly (i.e., change in wind pattern) and rely on other sensory information such as visual or tactile feedback. The following section provides an overview of the system proposed in this work and the general setup of the performed experiments.

\section{System architecture}

This work examines and combines known forms of sensory-assisted navigation, (i.e., the olfactory and tactile cues) in a novel arrangement in order to solve the problem of odor source localization. The vehicle designated to navigate in the environment is based on currently available unmanned ground vehicles (UGVs). Although car-sized UGVs use Ackermann steering such as their manned counterparts, smaller UGVs (i.e., less than $1 \mathrm{~m}$ in length) are constructed using differential steering. The proposed architecture (see Fig. 1) can be divided in two main components.

Firstly, the haptic device developed in [55] is set up to function as the UGV controller, two mechanisms of the device handle the robot tracks independently, in a system that is analogous to the thrust levers found in old tanks and tractors (see Fig. 2a). Each lever in turn provides force feedback to the human operator. The force algorithm is based on the perceived odor direction and is thoroughly explained in the following section. The position of the

Fig. 1 Schematic representation of the systems involved in the proposed model

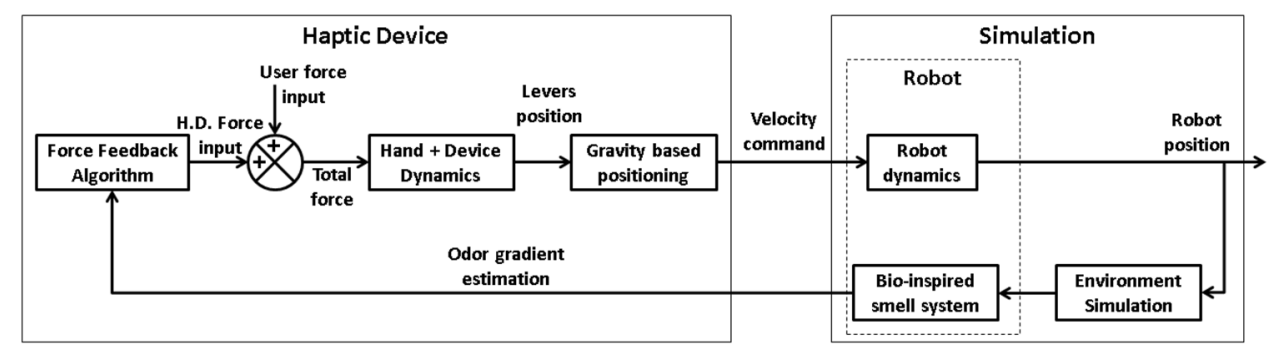

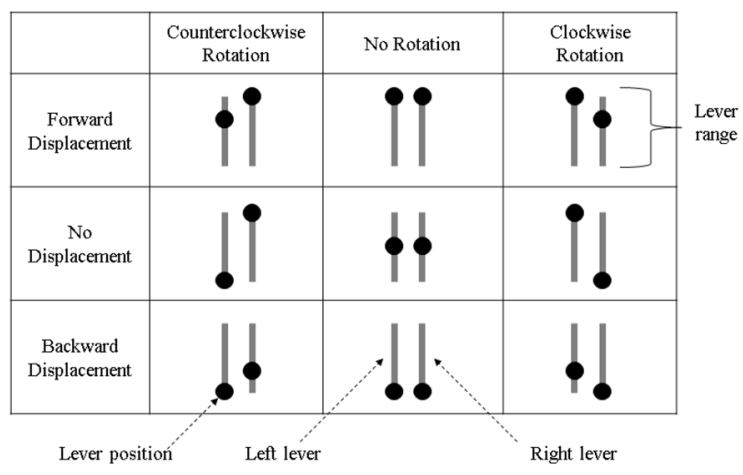

(a)

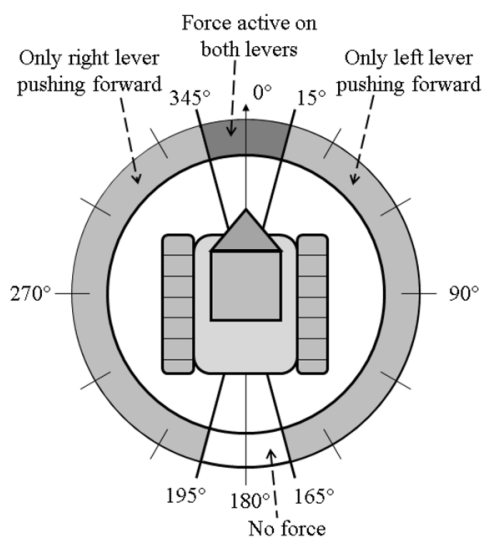

(b)
Fig. 2 Effect of user input on the UGV movement as function of the position of the haptic interface levers (a). Three rotation scenarios (clockwise, counterclockwise, and no rotation) and three translational displacement (forward, backward, and no displacement) yield nine possible movement combinations. Furthermore, force feedback as a function of the perceived direction of the odor source (b). Four possible actions are defined, if the odor is per- ceived to be on the front $\left( \pm 15^{\circ}\right)$, and both levers of the controller are pushed forward by the algorithm, inducing the user to advance faster. Conversely, if the odor is estimated to proceed from the rear (between $-165^{\circ}$ and $195^{\circ}$ ), both levers are inactivated. Odor perceived from either side activates a single lever in order to steer the robot toward the source (i.e., odor coming from the left activates a force pushing the right lever forward) 
levers is estimated by accelerometers, whose functioning is described in [55]. It depends on both the force feedback and the user input force. This position determines the velocity of each of the robot tracks (see Fig. 2b). The second component of the system is the simulated environment, which comprises the chemical dispersion, the UGV, and its attached smell system. The robot velocity is calculated using the commands sent from the haptic device. The UGV position and the corresponding chemical signatures in their sensors are used to estimate the orientation of the odor gradient. These data are used to calculate the haptic feedback sent to the user. Moreover, the simulated environment is programmed to mimic either idle or windy conditions in a designated area. The chemical simulation, robot position, and force feedback commands update at a rate of $10 \mathrm{~ms}$ to provide a seamless experience [35]. Human interacting interfaces used in this work include a force feedback device (Fig. 3) and a visual HMI (Fig. 4). The former is detailed in the following section, while the latter is explained as part of the simulation section.

\subsection{Haptic device}

The kinematic multipoint haptic device used in this research (see Fig. 3) has been comprehensively analyzed in previous work $[54,56]$. Furthermore, this device has been used to control an UAV by a human operator, where the implemented software for this application diverted the UAV trajectory in case of an impending obstacle collision [53]. Five seven-bar mechanisms comprise the apparatus, each with two degrees of freedom (DOFs) and the corresponding number of motors. For this application, only a pair of mechanisms are used (see Fig. 3b). The motors can deliver up to $0.2 \mathrm{Nm}$ when stalled at full voltage; the continuous use of the motor at this configuration, however, leads to damage of the device. Therefore, the system is controlled at 3 volts (V), drawing 3 amperes (A), and producing a $0.05 \mathrm{Nm}$ torque. This torque is transmitted to the upper bar using a toothed belt with a speed ratio of 2:1. Given the motor, belt transmission, and the $40 \mathrm{~mm}$ bar, the estimated force applied to the user is of $2.5 \mathrm{~N}$, which is sufficient to be perceived by the user [19] but less than the typical force that an individual can exert [18]. The angular displacement of the upper bar translates into the corresponding track velocity. Moreover, the angular displacement of the lower bar does not affect the signal and the user can accommodate this kinematic pair to maximize comfort (see Fig. 3). The haptic device design allows the user to rest their hands on top of the motor casing in order to grab the mechanism without inducing fatigue. Furthermore, the experimental design limits the maximum time an individual can be operating the device up to thirty minutes. This protects both the individual and the experimental devices from any fatigue or overheatingrelated damage, respectively. The angular displacement of the mechanism kinematic pairs is continually measured using accelerometers, which represent an improvement over encoders that require additional mechanical components, cause undesired friction, and have moving parts that require maintenance after prolonged use. A 10-bit analog-to-digital converter (ADC) and the abovementioned accelerometers allow sub-degree resolution, which detects the position of the mechanism end effector within fractions of a millimeter; this feature can be further enhanced with more precise ADCs (see [55]). The microcontroller ADC transduces and transmits the accelerometers analog signal to a PC where the driver interacts with the simulation and its corresponding $\mathrm{HMI}$, which are thoroughly explained in the following section.
Fig. 3 Basic 2-DOF mechanism of the haptic device used in the experiments (a). Two of the five mechanism of the apparatus are used in this work; the authors encouraged but not forced the operators to adopt the shown hand posture (b)

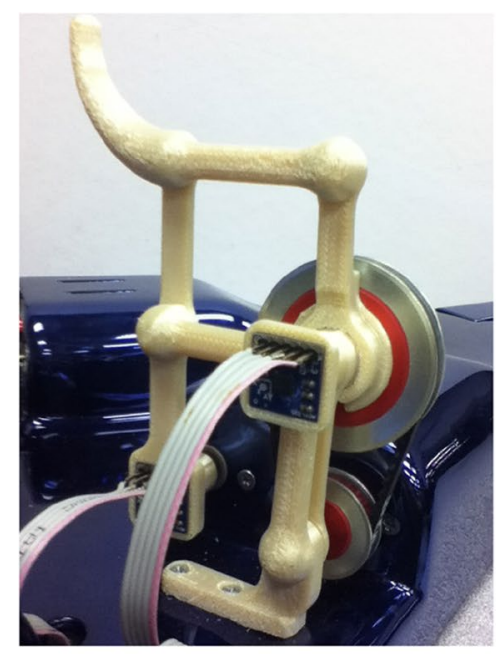

(a)

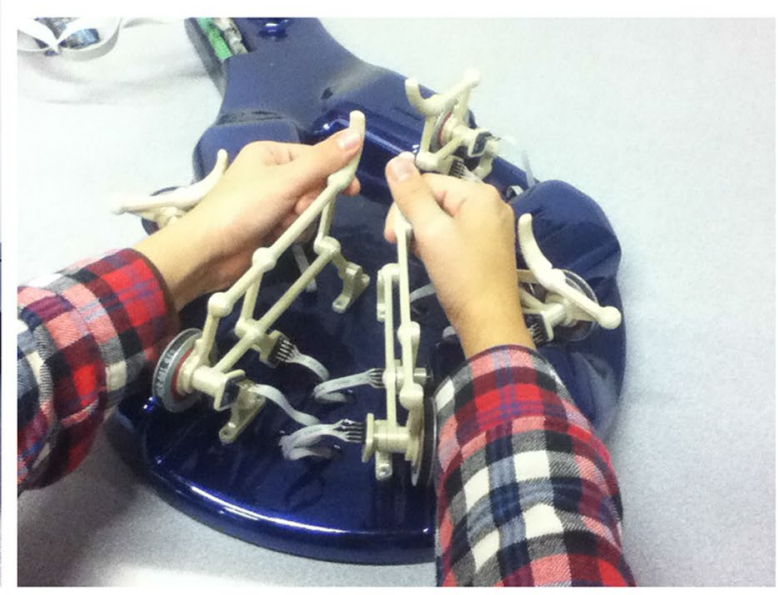

(b)

SN Applied Sciences A SPRINGER NATURE journa 


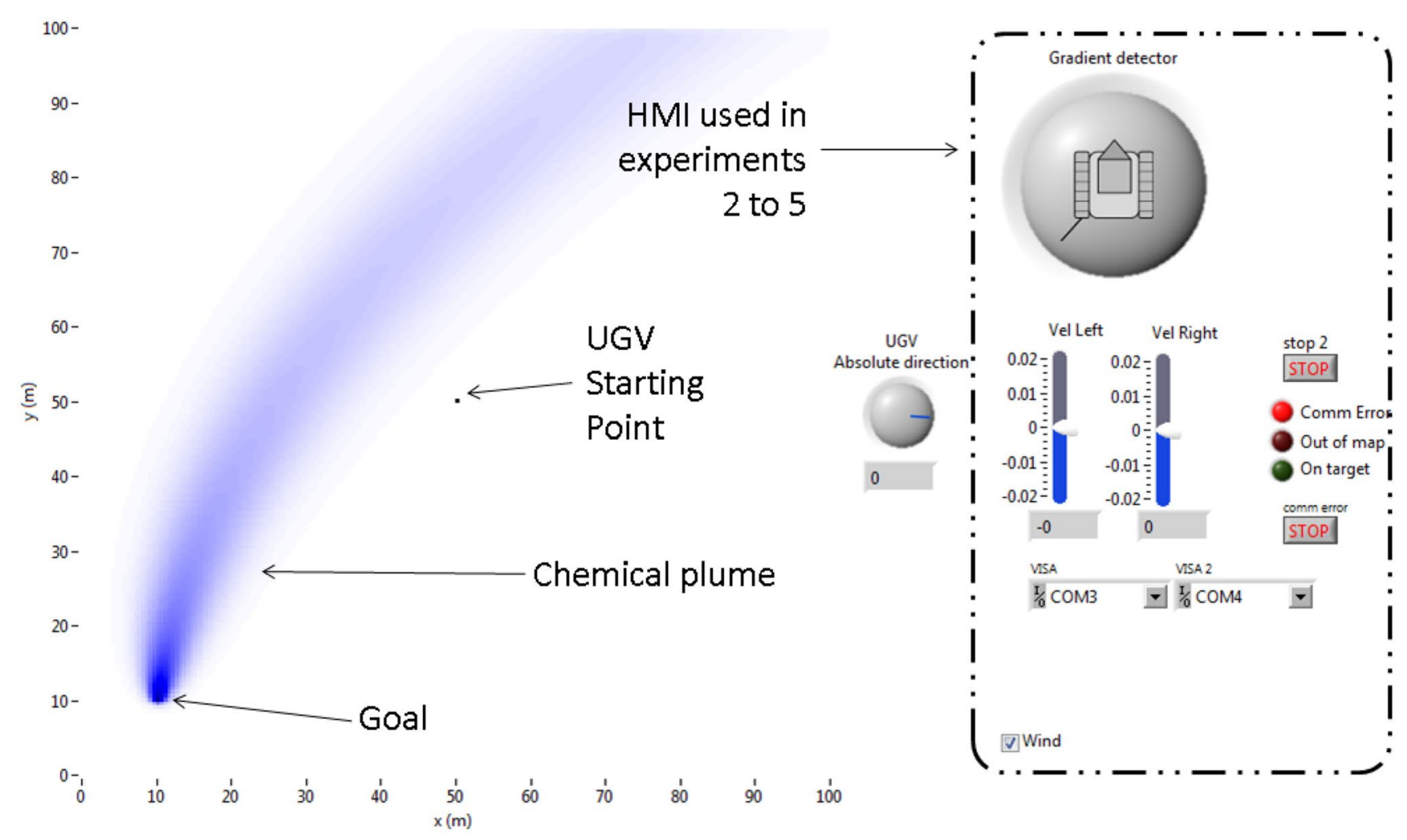

Fig. 4 Human-machine interface as seen by the user in experiment 1 and denoted as $A+$ in Table 1. The dotted line encloses the region available to the user in experiments 2 to 5 and depicted as $A$ in the

above-mentioned table. The gradient detector is a knob with a line that points to the gradient direction estimated by the sensors and referenced in respect of the front face of the UGV

\subsection{Simulation}

This work simulates a 20 -cm-long UGV with independent track motion. The operators are capable to operate the device using the levers mentioned in the previous section, which control the UGV corresponding track independently. The UGV maximum speed is set to be 0.5 $\mathrm{m} / \mathrm{s}$. The relative size of the map and the robot does not allow the latter to be properly seen; therefore, a dial is set right to the emap in order to show the robot orientation at any given time (see Fig. 4). Furthermore, the vehicle is equipped with odor sensors capable of detecting chemical gradients. Note that sensors found in the literature $[39,42]$ present limitations such as detectability thresholds highly dependent on the gradient angle relative to the vehicle. This work, however, supposes that the concentrations the vehicle encounters always fall within the sensor perception range. It is also assumed that the chemical detectors are omnidirectional and therefore present ideal directivity.

To thoroughly test the hypothesis that chemical information can be transmitted to a human operator, a simulation environment is designed using LabVIEW. The simulation is bounded to a $100 \mathrm{~m} \times 100 \mathrm{~m}$ area in which a point source of odor exists; this size discards the arrival to the

odor source by chance. In a real environment, such chemical would disseminate by multiple, complex mechanisms such as conversion, pressure and temperature differentials, laminar and turbulent currents, eddies, wind vortices, and Brownian motion, all of which have been studied in the last decades. For the presented simulation, however, the dispersion mechanisms are simplified in two principal components: firstly by diffusion considering it ideal and secondly by advection which corresponds to the effect of currents within the simulated environment, both thoroughly explained in this work ((3)). Four of the seven experiments presented to each operator include a current whose direction and magnitude vary as a function of position and set to be up to $2.5 \mathrm{~m} / \mathrm{s}$. In all performed experiments, diffusion is present; in experiments 4, 5, and 7, however, wind dominates over diffusion by shaping a distinct distribution pattern (see Fig. 5d-f), unlike experiments 1, 2, 3, and 6 where diffusion solely determines the chemical distribution (see Fig. 5a-c). Note that the chemical dispersion evolves in time, but converges to a steady state for either quiescent or windy conditions as shown in Fig. $5 c$, f, respectively. The next section explains these experimental variations, their starting conditions, and the different types of feedback that the operators will encounter. 
Table 1 Parameter disposition in the experiments

\begin{tabular}{|c|c|c|c|c|c|c|c|c|c|}
\hline \multirow[t]{2}{*}{ Experiment } & \multirow[t]{2}{*}{ Wind } & \multirow[t]{2}{*}{ Force } & \multirow[t]{2}{*}{ Graphics } & \multicolumn{3}{|l|}{ Time } & \multicolumn{3}{|c|}{ Distance } \\
\hline & & & & $\mu(\mathrm{s})$ & $\sigma(\mathrm{s})$ & KS test & $\mu(\mathrm{m})$ & $\sigma(\mathrm{s})$ & $\mathrm{KS}$ test \\
\hline 1 & $\mathrm{D}$ & $A$ & $A+$ & 65.85 & 7.093 & 0.6029 & 67.20 & 6.99 & 0.326 \\
\hline 2 & $\mathrm{D}$ & $\mathrm{D}$ & A & 82.93 & 14.65 & 0.9577 & 66.46 & 4.41 & 0.785 \\
\hline 3 & $\mathrm{D}$ & A & A & 64.21 & 7.97 & 0.4215 & 65.83 & 5.37 & 0.241 \\
\hline 4 & A & $\mathrm{D}$ & A & 120.36 & 24.41 & 0.9872 & 92.98 & 9.28 & 0.899 \\
\hline 5 & A & A & A & 100.76 & 17.94 & 0.3419 & 90.51 & 11.30 & 0.153 \\
\hline 6 & $\mathrm{D}$ & A & $\mathrm{D}$ & 69.69 & 12.87 & 0.2977 & 70.45 & 12.47 & 0.214 \\
\hline 7 & A & A & $\mathrm{D}$ & 112.48 & 26.39 & 0.5748 & 100.56 & 17.69 & 0.155 \\
\hline ALL & - & - & - & 88.04 & 27.46 & - & 79.14 & 17.30 & - \\
\hline
\end{tabular}

$D$ deactivated, $A$ activated. $A+$ denotes the additional visual information provided to the user in experiment 1

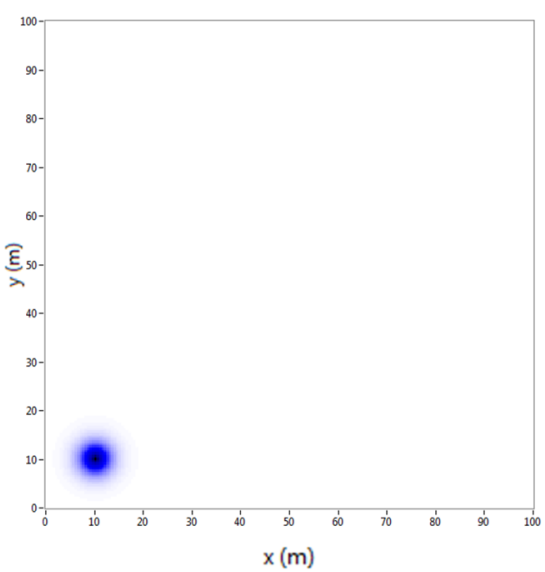

(a)

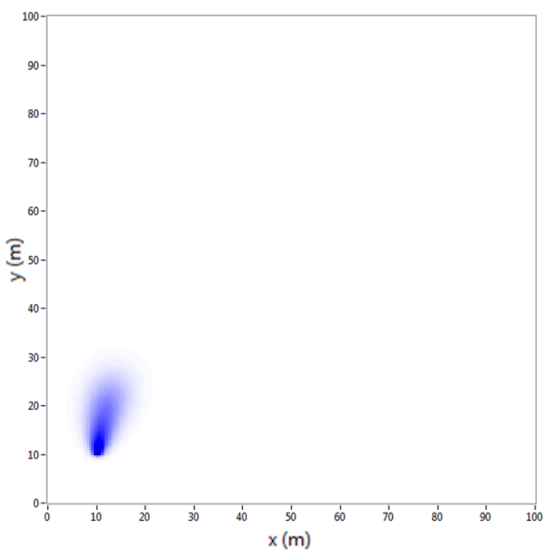

(d)

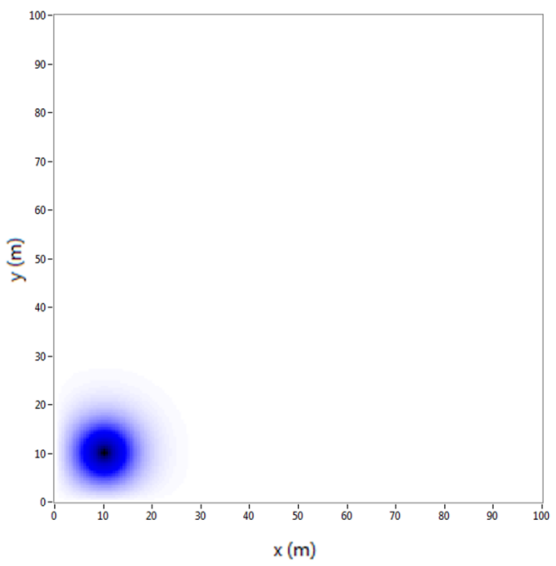

(b)

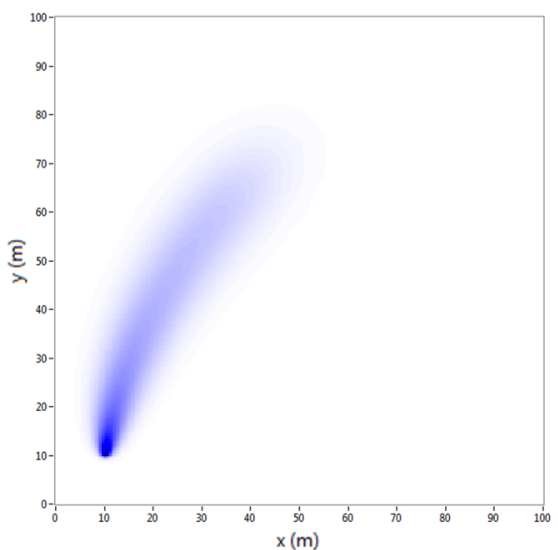

(e)

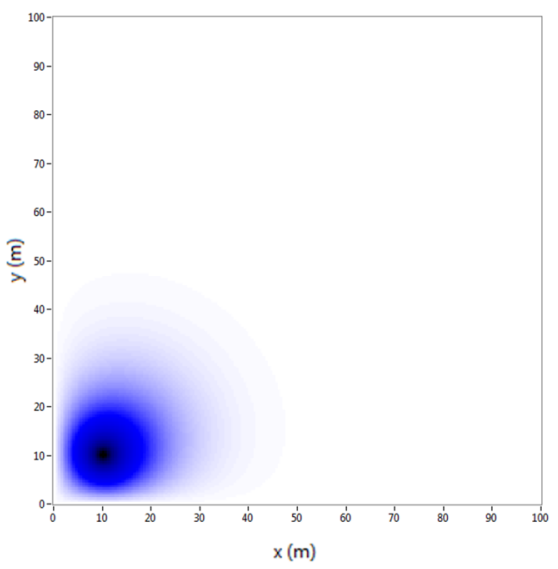

(c)

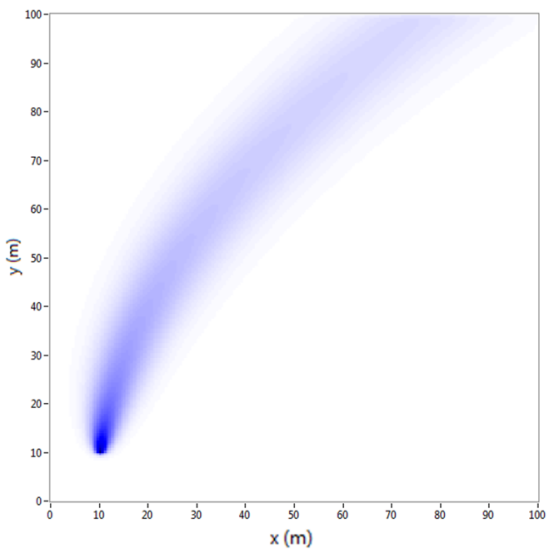

(f)

Fig. 5 Evolution of odor dispersion in the 2D environment. Representative frames are captured at 10s, 40s, and 200s for idle $\mathbf{a}, \mathbf{b}$, and $\mathbf{c}$ and windy $\mathbf{d}, \mathbf{e}$, and $\mathbf{f}$ conditions

\section{Experimental work and results}

Twenty-four users, aged between 23 and 35, participated in the experiments. Each experimental run is comprised by seven experiments that accrue into one hundred sixty-eight individual experiments. The tests occur in the simulated environment, where various parameters are modified, namely the wind currents, 
the haptic feedback, and the amount of visual feedback available.

As an introductory test, novice operators are initially allowed to navigate in the simulated environment and interact with all available visual and tactile feedback. A proper explanation is given about the nature of the levers that control the robot, the force feedback experienced, and the instruments that the user can access through the HMI. The introductory simulation is restarted if the user abandons the map. This introductory part of the experiment lasts until the user expresses its confidence to continue into the timed trials. Once the introduction is complete, the user navigates the environment in seven distinct experiments, all of which start and finish at the coordinates $(50,50)$ and $(10,10)$, respectively. Furthermore, the robot orientation starts pointing on the positive $X$-axis. The experiments end when the user is at $5 \mathrm{~m}$ or less of the target; this condition is informed to the driver either through the HMI as shown in Fig. 4 or verbally in the experiments with no visual feedback. At this distance, a robot can easily find the odor source autonomously by means of different algorithms [62]. The sequence of experiments, specially the introductory test and the first experiment, was designed to habituate the operator to the device and feedback behavior. Furthermore, for a given set of visual or haptic feedback, the experiment with no air flow precedes the experiment with the current activated.

In the first experiment, which works as a control and further reinforces the operator learning process, the individual navigates in an environment with no wind currents (see Fig. $5 \mathrm{a}-\mathrm{c}$ ). The user navigates using force feedback of the haptic device and visual feedback from the screen, from which it can see both the entire map and the estimated direction of the chemical trail as shown in Fig. 4 and denoted as A + in Table 1. The second experiment also operates with no airflow, force feedback is turned off and the visual feedback is limited (see dashed line in Fig. 4) to the gradient detector (the line departing from the robot), the levers position, and the signaling lights for the special conditions. These lights signal if the user reaches the target navigates out of the map boundaries or if an error occurs in the communication between the computer and the haptic device. The third experiment is a reiteration of the second, except for the force feedback feature, which is switched on. The time to reach the objective by the user can be compared with the previous experiment in order to assess the effect of the force feedback in the driver behavior.

For the fourth experiment, the force feedback is deactivated and air currents are added to the simulation. The wind generates a chemical trail as depicted in Fig. $5 f$. Note that the wind speed and direction are not constant throughout the working area; however, its magnitude has been set to be lower than $2.5 \mathrm{~m} / \mathrm{s}$. Similarly to the third experiment, the fifth is a repetition of the previous trial with force feedback activated in the haptic device. The time necessary to reach the objective is expected to increase in the experiments where air currents exist, as the variable measured from the environment becomes more convoluted. The sixth experiment has the air currents and visual feedback deactivated, while the user relies only in haptic feedback to reach the objective, where the success of this test shows the ability of haptic feedback to provide meaningful information in navigation applications. Moreover, the time it takes to complete this experiment can be compared with previous trials in order to support or reject the idea that tactile feedback enhances the operators performance over visual information. For the seventh and last experiment, force feedback remains as the only source of information and air currents are activated to increase the setup difficulty and assess the capability of force to ensure successful navigation toward the target. The seven experiments and their corresponding characteristics are given in Table 1. Wind, visual feedback, and force feedback are the parameters altered in the mentioned experiments, they can be either activated or deactivated and these setups are encoded as $A$ or $D$, respectively. The first experiment has additional visual feedback, marked as $A+$ in Table 1 , where the operator is also shown the map overview as shown in Fig. 4. Table 1 also depicts statistical information of the experiments, namely the mean time to reach the target $\mu$ and the corresponding standard deviation $\sigma$. Further statistical information is presented in the boxplot of Fig. 6. Note that Table 1 and the boxplot in Fig. 6 portray the mean and the median, respectively.

The resulting trajectories of the twenty-four runs of each experiment are depicted in Figs. 7 and 8. The results show a characteristic difference between trials with and without air currents. While the experiments without wind tend to follow a straight path from the start to the finish points, the experiments with activated air current exhibit two distinctive sections. The starting segment flows not to the finish point, but to the chemical trail. The second section slides through the chemical trail until the robot reaches the target. Note that the path through the chemical trail is not rectilinear, but wobbly as the perceived direction of the odor source changes with small displacements. A control algorithm that mitigates this unsteady behavior should reduce the time to reach the target and prove useful in chemotaxis-based navigation applications.

Every run took between 54.1 and $176.74 \mathrm{~s}$ to reach the target, with an average $\mu=88.04 \mathrm{~s}$ and a standard deviation $\sigma=27.46 \mathrm{~s}$. Experiments 1 and 3 that have visual and tactile feedback have resulted in the shortest finishing times ( $\mu_{1}=65.85 \mathrm{~s}$, and $\left.\mu_{3}=64.21 \mathrm{~s}\right)$, and experienced the 


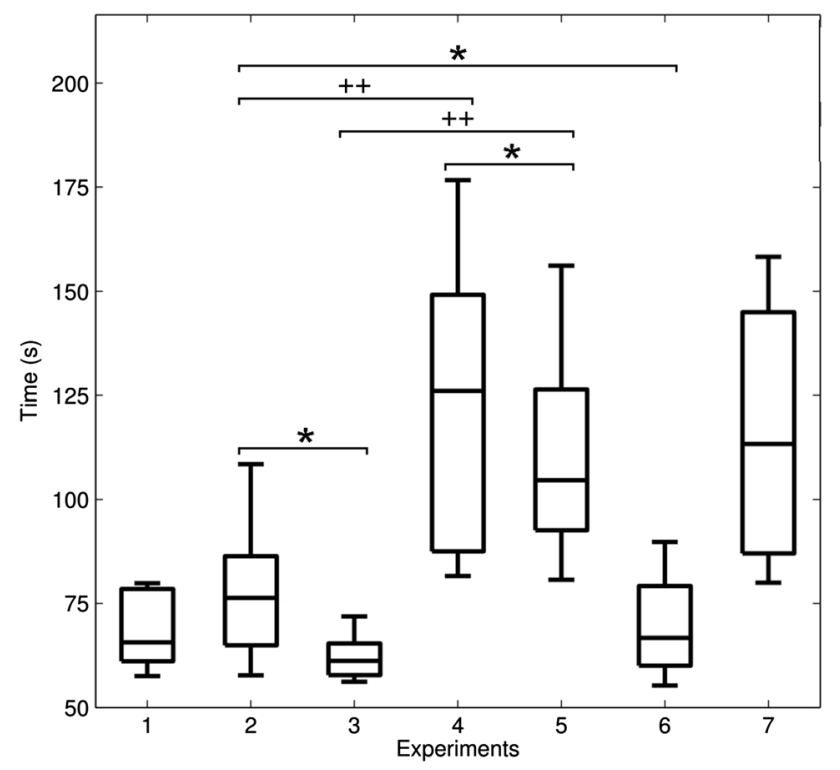

Fig. 6 Time distribution the seven experiments. Haptic feedback reduces arrival time if other features in the experiment remain equal as seen in experimental pairs 2-3 and 4-5. All experimental pairs with statistical significant differences mentioned in this work are depicted with one asterisk $\left(^{*}\right)$ or two crosses $(++)$ for $p<0.001$ and $p<0.0001$, respectively

smallest standard deviations $\left(\sigma_{1}=7.093 \mathrm{~s}\right.$, and $\sigma_{3}=7.973$ s). Conversely, experiments 4 and 7 retained the longest average times ( $\mu_{4}=120.36 \mathrm{~s}$, and $\mu_{7}=112.48 \mathrm{~s}$ ) and the highest standard deviations $\left(\sigma_{4}=24.41 \mathrm{~s}\right.$, and $\sigma_{7}=26.39$ $\mathrm{s})$; these experiments are characterized by having windy conditions and only a single source of information, either tactile or visual.

The following statistical analysis uses experimental pairs to draw conclusions based on the obtained distributions. The Student's $t$, Mann-Whitney $U$, and ANOVA tests were considered for statistical analysis. ANOVA is the preferred option for test several groups as long as normality and similar variance can be assumed. Note that the Mann-Whitney $U$ test does not require the assumption of normality. Similarly, note that the Student's $t$ test can work with unequal variances. The resulting data present close to normal distributions and unequal variances (see Table 1). For this reason, the null hypothesis $\mathrm{H}_{0}$ that a given pair of experiments have the same distribution is rejected or retained using the Student's $t$ test. Furthermore, the Kolmogorov-Smirnov test (KS test) supports the assumption of data normality required by the Student's $t$ test. The seven data sets yield values higher than the typical $(p<$ 0.05 ) that would reject the null hypothesis that the data sets follow a normal distribution (see Table 1).

The most significant difference between experiments arises by the existence of air currents, which vastly increase the time the user takes to reach the finish point. This can be observed in experimental pairs 2-4 (no haptic feedback and graphics partially activated) and 3-5 (haptic feedback activated and graphics partially activated), where the presence of wind delays the finishing with average times of 37.43 and $36.55 \mathrm{~s}$ that have a strong statistical significance $(p<0.0001)$. Force feedback reduces the time to reach the objective if other parameters remain identical. For experimental pairs 2-3 (no wind and graphics partially activated) and $4-5$ (wind activated and graphics partially activated), the activation of force feedback reduces the average time to reach the target 18.72 and $19.60 \mathrm{~s}$, respectively $(p<$ 0.001 ); this shows a favorable effect of transferring navigational information through tactation even if the same instructions are transmitted using audio-visual means. Furthermore, solely haptic feedback yields better performance than solely visual feedback as shown in experimental pair 2-6 (no wind) with an average reduction of 13.24 $\mathrm{s}(p<0.001)$.

\section{Discussion}

A force feedback device has been successfully used in assisted navigation based on chemotaxis for odor source localization. A simulated environment was created to run seven experiments, three of which were designed with air currents and the rest in stagnant conditions. The $100 \mathrm{~m} \times$ $100 \mathrm{~m}$ simulated environment included a chemical source that is used as the destination; all experiments start at the center of the map. One hundred sixty-eight experiments, comprised by seven distinct scenarios, were performed by twenty-four users. Operators navigated in the simulated environment with the instruction to reach the odor source. Each individual repeated the setup in seven different configurations. All operators arrived at the desired location in every experiment regardless of these changes in the environment and the use of different sources of information, which include only tactile, only visual, or a combination. This work represents a natural extension from autonomous algorithms that are used in several applications, but lack the human natural ability to adapt to complex environments. This ability is critical in the variations produced by wind currents, and further work regarding chemotaxisbased autonomous navigation should focus on the algorithm robustness under varying conditions.

The results show that wind currents affect significantly the results, which should be taken into account in future research toward new devices and algorithms and their performance in field experimentation; if a given setup underperforms, it may have to do more with changes in wind patterns and less with the algorithm. The 


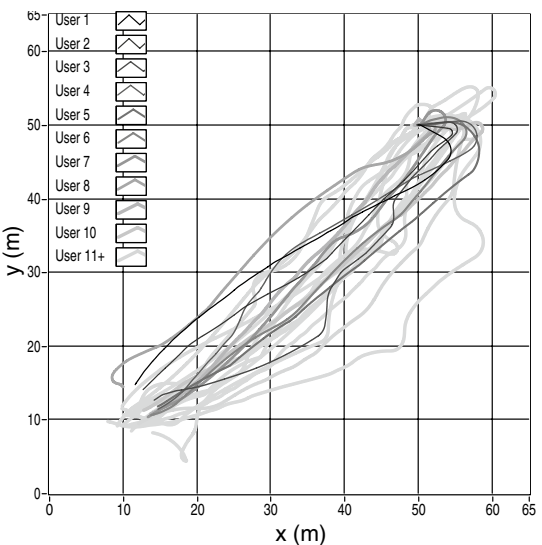

$\operatorname{Exp} 1$, Wind $=\mathrm{D}$, Force $=\mathrm{A}$, Graphics $=\mathrm{A}+$

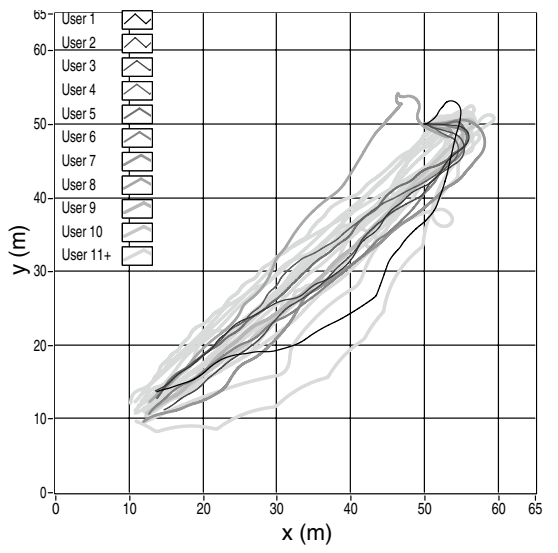

Exp 2, Wind $=\mathrm{D}$, Force $=\mathrm{D}$, Graphics $=\mathrm{A}$

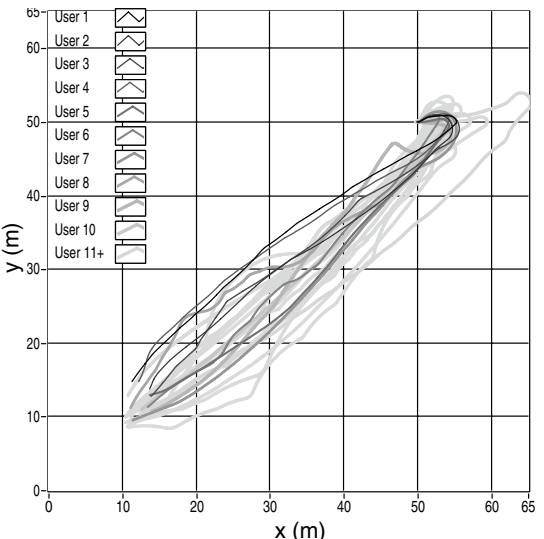

Exp 3, Wind $=\mathrm{D}$, Force $=\mathrm{A}$, Graphics $=\mathrm{A}$

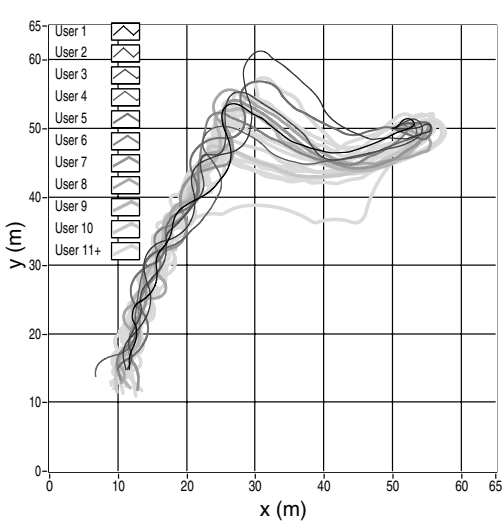

$\operatorname{Exp} 4$, Wind $=\mathrm{A}$, Force $=\mathrm{D}$, Graphics $=\mathrm{A}$

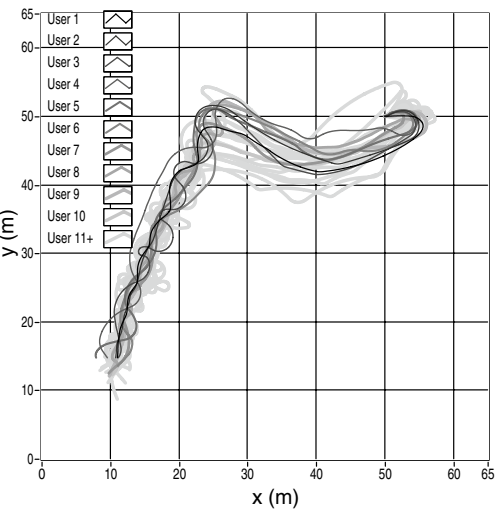

$\operatorname{Exp} 5$, Wind $=\mathrm{A}$, Force $=$ A, Graphics $=$ A

Fig. 7 Resulting trajectories in experiments 1 through 5. D Deactivated, A Activated. A + denotes the additional visual information provided to the user in experiment 1

experiments confirm related work that haptic feedback can have an important role in assisted navigation applications. Moreover, haptic feedback enhances navigational performance in environments where the operator originally relied solely on visual information; this particular result has relevant repercussions in multiple navigation applications. If a person responds better to haptic cues, preventable errors in automotive accidents or flawed surgical procedures could be avoided with additional haptic feedback.

Furthermore, the experiment compares situations where the user receives either only-touch or only-visual cues and demonstrates enhanced performance where haptic feedback replaces visual information. The setup simplicity enables users to use the system effectively with a expeditious learning process. This shows the natural human ability to navigate using tactile feedback with little or no training, representing a topic with potential for analysis in future work. Potential applications of this innate ability could be used in training and rehabilitation applications. This work uses one haptic device with one force feedback algorithm, and further work could perform similar experiment with different algorithms and devices to assess changes in effectiveness and efficiency. A thorough study of various wind patterns can elucidate the limits in which chemotaxis allows successful navigation toward an odor source. Experimentation not presented in this document showed that alternating wind patterns increase the difficulty for drivers to reach an odor source. Therefore, the authors recommend that future work encompasses various wind velocities and alternating patterns of idle and windy conditions. 


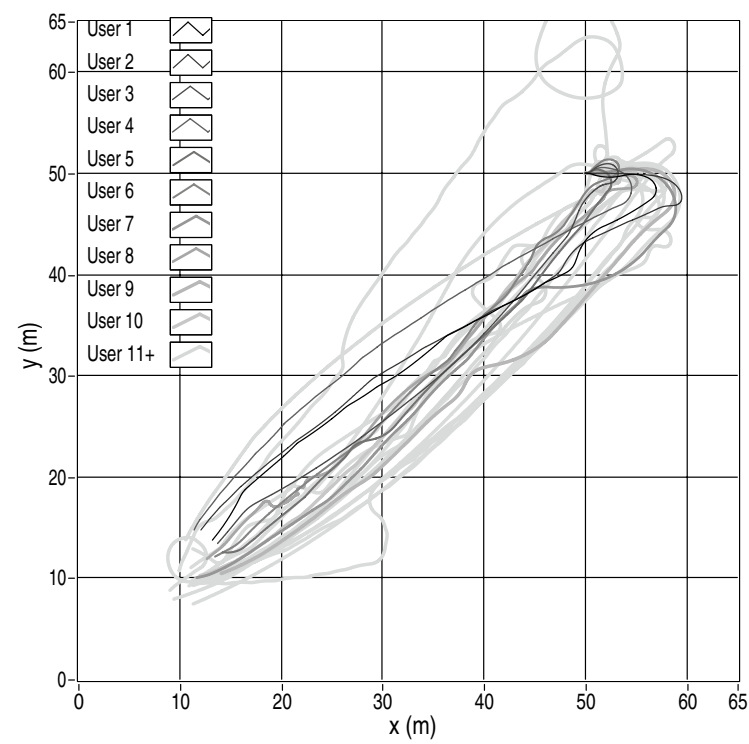

Exp 6, Wind $=\mathrm{D}$,

Force $=$ A, Graphics $=$ D

Fig. 8 Resulting trajectories in experiments 6 and 7

Acknowledgements The authors would like to acknowledge Consejo Nacional de Ciencia y Tecnologia (CONACyT), the e-Robots Research Chair from Tecnologico de Monterrey, Campus Monterrey, and the Laboratorio de Robotica del Area Noreste y Centro de Mexico for supporting this research.

Funding This study was funded by Tecnologico de Monterrey and Consejo Nacional de Ciencia y Tecnologia (CONACyT).

\section{Compliance with ethical standards}

Conflict of interest The authors declare that they have no conflict of interest.

\section{References}

1. Ackerman E (2015) Unlucky robot gets stranded inside fukushima nuclear reactor, sends back critical data. IEEE Spectrum 20

2. Aggravi M, Pause F, Giordano PR, Pacchierotti C (2018) Design and evaluation of a wearable haptic device for skin stretch, pressure, and vibrotactile stimuli. IEEE Robot Autom Lett 3(3):21662173. https://doi.org/10.1109/LRA.2018.2810887

3. Aggravi M, Scheggi S, Prattichizzo D (2015) Evaluation of a predictive approach in steering the human locomotion via haptic feedback. In: 2015 IEEE/RSJ international conference on intelligent robots and systems (IROS), pp 597-602. https://doi. org/10.1109/IROS.2015.7353433

4. Agrawal S, Chen X, Ragonesi C, Galloway J (2012) Training toddlers seated on mobile robots to steer using force-feedback

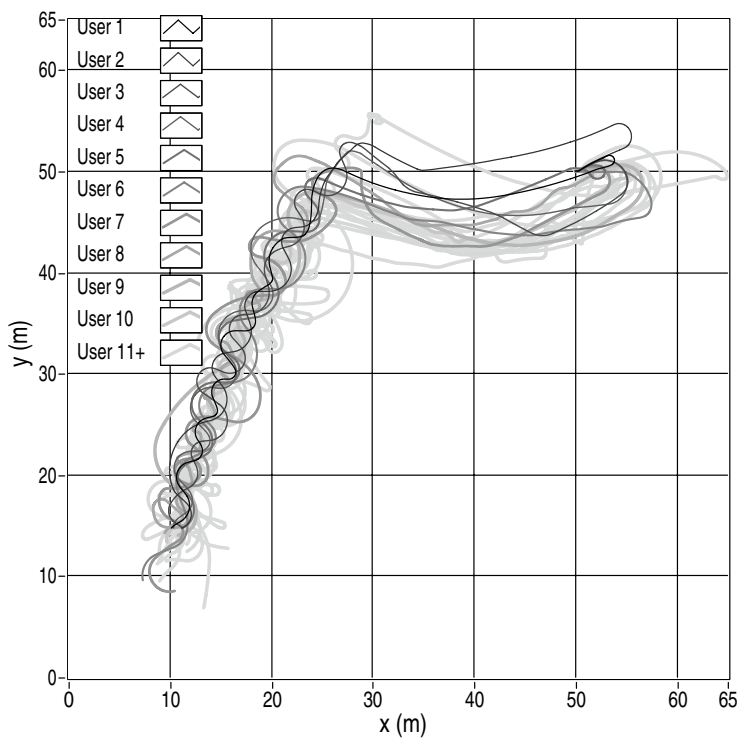

$\operatorname{Exp} 7$, Wind $=\mathrm{A}$,

Force $=$ A, Graphics $=$ D joystick. IEEE Trans Haptics 5(4):376-383. https://doi. org/10.1109/TOH.2011.67

5. Ahmaniemi T, Marila J, Lantz V (2010) Design of dynamic vibrotactile textures. IEEE Trans Haptics 3(4):245-256. https://doi. org/10.1109/TOH.2010.22

6. Arbabtafti M, Moghaddam M, Nahvi A, Mahvash M, Richardson B, Shirinzadeh B (2011) Physics-based haptic simulation of bone machining. IEEE Trans Haptics 4(1):39-50. https://doi. org/10.1109/TOH.2010.5

7. Asque C, Day A, Laycock S (2014) Augmenting graphical user interfaces with haptic assistance for motion-impaired operators. Int J Human-Comput Stud 72(1011):689-703. https://doi. org/10.1016/j.ijhcs.2014.05.007

8. Baldi TL, Scheggi S, Aggravi M, Prattichizzo D (2018) Haptic guidance in dynamic environments using optimal reciprocal collision avoidance. IEEE Robot Autom Lett 3(1):265-272. https:// doi.org/10.1109/LRA.2017.2738328

9. Boessenkool H, Abbink D, Heemskerk C, van der Helm F, Wildenbeest J (2013) A task-specific analysis of the benefit of haptic shared control during telemanipulation. IEEE Trans Haptics 6(1):2-12. https://doi.org/10.1109/TOH.2012.22

10. Bren A, Eisenbach $M(2000)$ How signals are heard during bacterial chemotaxis: protein-protein interactions in sensory signal propagation. J Bacteriol 182(24):6865-6873

11. Cabrita G, Sousa P, Marques L (2011) Odor guided exploration and plume tracking: Particle plume explorer. In: Proceedings of the 5th european conference on mobile robots (ECMR 2011), pp $165-170$

12. Catania K, Remple F (2004) Tactile foveation in the star-nosed mole. Brain Behav Evol 63(1):1-12

13. Chang W, Hwang W, Gu Y (2011) Haptic seat interfaces for driver information and warning systems. Int J Human-Comput Interact 27(12):1119-1132. https://doi.org/10.1080/10447 318.2011 .555321 
14. Chapwouo Tchakouté LD, Gagnon D, Ménélas BAJ (2018) Use of tactons to communicate a risk level through an enactive shoe. J Multimodal User Interfaces 12(1):41-53. https://doi. org/10.1007/s12193-018-0260-8

15. Coles T, Meglan D, John N (2011) The role of haptics in medical training simulators: a survey of the state of the art. IEEE Trans Haptics 4(1):51-66. https://doi.org/10.1109/TOH.2010.19

16. Craighead $F$ (1976) Grizzly bear ranges and movement as determined by radiotracking. Bears: Their Biol Manag 3:97-109

17. Crank J (1975) The mathematics of diffusion, $2 d$ edn. Clarendon Press, Oxford

18. Didomenico A, Nussbaum M (2003) Measurement and prediction of single and multi-digit finger strength. Ergonomics 46(15):1531-1548. https://doi.org/10.1080/001401303200012 1660 PMID: 14668173

19. Dosher J, Hannaford B (2005) Human interaction with small haptic effects. Presence 14(3):329-344. https://doi. org/10.1162/105474605323384672

20. Elliott L, van Erp J, Redden E, Duistermaat M (2010) Field-Based Validation of a Tactile Navigation Device. IEEE Trans Haptics 3(2):78-87. https://doi.org/10.1109/TOH.2010.3

21. Endo T, Kawasaki H (2015) A fine motor skill training system using multi-fingered haptic interface robot. Int J Human-Comput Stud 84:41-50. https://doi.org/10.1016/j.ijhcs.2015.08.006

22. Estakhroyeh HR, Rashedi E, Mehran M (2018) Design and construction of electronic nose for multi-purpose applications by sensor array arrangement using ibgsa. J Intell Robot Syst 92(2):205-221. https://doi.org/10.1007/s10846-017-0759-3

23. Fischer $H$ (1979) Mixing in inland and coastal waters. Academic press, Edinburgh

24. Francis KB, Terbeck S, Briazu RA, Haines A, Gummerum M, Ganis G, Howard IS (2017) Simulating moral actions: An investigation of personal force in virtual moral dilemmas. Sci Rep 7(1):13954

25. Gibson C (2010) Hormones and behaviour: a psychological approach (review). Perspect Biol Med 53(1):152-155

26. Illanes A, Boese A, Maldonado I, Pashazadeh A, Schaufler A, Navab N, Friebe M (2018) Novel clinical device tracking and tissue event characterization using proximally placed audio signal acquisition and processing. Sci Rep 8(1):12070

27. Ishida $\mathrm{H}$, Tanaka $\mathrm{H}$, Taniguchi $\mathrm{H}$, Moriizumi $\mathrm{T}$ (2006) Mobile robot navigation using vision and olfaction to search for a gas/odor source. Auton Robot 20(3):231-238

28. Jørgensen TB, Jensen SHN, Aanæs $H$, Hansen NW, Krüger N (2018) An adaptive robotic system for doing pick and place operations with deformable objects. J Intell Robot Syst https:// doi.org/10.1007/s10846-018-0958-6

29. Katzschmann RK, Araki B, Rus D (2018) Safe local navigation for visually impaired users with a time-of-flight and haptic feedback device. IEEE Trans Neural Syst Rehabil Eng 26(3):583-593. https ://doi.org/10.1109/TNSRE.2018.2800665

30. Kawasaki H, Ohtuka Y, Koide S, Mouri T (2011) Perception and haptic rendering of friction moments. IEEE Trans Haptics 4(1):28-38. https://doi.org/10.1109/TOH.2010.42

31. Kawatsuma S, Fukushima M, Okada T (2012) Emergency response by robots to fukushima-daiichi accident: summary and lessons learned. Ind Robot: Int J 39(5):428-435

32. Kowadlo G, Russell A (2008) Robot odor localization: a taxonomy and survey. Int J Robot Res 27(8):869-894

33. Lam T, Boschloo H, Mulder M, Van Paassen M (2009) Artificial force field for haptic feedback in uav teleoperation. IEEE Trans Syst Man Cyber Part A Syst Humans 39(6):1316-1330. https:// doi.org/10.1109/TSMCA.2009.2028239

34. Larcombe M, Halsall J (1984) Robotics in nuclear engineering: computer-assisted teleoperation in hazardous environments with particular reference to radiation fields, vol. 9312. Graham \& Trotman
35. Lederman S, Jones L (2011) Tactile and haptic illusions. IEEE Trans Haptics 4(4):273-294. https://doi.org/10.1109/TOH.2011.2

36. Lee D, Franchi A, Son HI, Ha C, Bulthoff H, Giordano P (2013) Semiautonomous haptic teleoperation control architecture of multiple unmanned aerial vehicles. IEEE/ASME Trans Mechatron 18(4):1334-1345. https://doi.org/10.1109/TMECH.2013.2263963

37. Lee S, Kim GJ (2008) Effects of haptic feedback, stereoscopy, and image resolution on performance and presence in remote navigation. Int J Human-Comput Stud 66(10):701-717. https:// doi.org/10.1016/j.ijhcs.2008.05.001

38. Lee S, Lee H, Lee I, Tseng C (2004) Ink diffusion in water. Eur J Phys 25(2):331-336

39. Lilienthal A, Duckett T (2003) A stereo electronic nose for a mobile inspection robot. In: 1st International Workshop on IEEE Robotic Sensing, 2003. ROSE'03, p 6

40. Lilienthal A, Duckett T (2004) Experimental analysis of gas-sensitive braitenberg vehicles. Adv Robot 18(8):817-834. https:// doi.org/10.1163/1568553041738103

41. Martinac B (2011) Bacterial mechanosensitive channels as a paradigm for mechanosensory transduction. Cell Physiol Biochem 28(6):1051-1060

42. Miyatani I, Ishida $H$ (2010) Active stereo nose: using air curtain to enhance the directivity. In: IEEE Sensors, pp 1522-1525

43. Myint M, Yonemori K, Lwin KN, Yanou A, Minami M (2018) Dualeyes vision-based docking system for autonomous underwater vehicle: an approach and experiments. J Intell Robot Syst 92(1):159-186. https://doi.org/10.1007/s10846-017-0703-6

44. Nakamoto $T$, Ishida $H$, Moriizumi $T$ (1996) An odor compass for localizing an odor source. Sens Actuators B: Chem 35(1):32-36. https://doi.org/10.1016/S0925-4005(96)02009-6

45. Nakamoto T, Ishida H, Moriizumi $T$ (1997) Active odor sensing system. ISIE '97 Proc IEEE Int Symp Ind Electron 1-3:128-133

46. Nakamoto T, Matsushita H, Okazaki N (1995) Improvement of optimization algorithm in active gas/odor sensing system. Sens Actuators A Phys 50(3):191-196

47. Nakamoto T, Okazaki N, Mornzumi T (1997) High speed active gas/odor sensing system using adaptive control theory. Sens Actuators B Chem 41:183-188

48. Nestmeyer T, Robuffo Giordano P, Bülthoff HH, Franchi A (2017) Decentralized simultaneous multi-target exploration using a connected network of multiple robots. Auton Robots 41(4):9891011. https://doi.org/10.1007/s10514-016-9578-9

49. Neumann P, Asadi S, Lilienthal A, Bartholmai M, Schiller J (2012) Autonomous gas-sensitive microdrone: wind vector estimation and gas distribution mapping. IEEE Robot Autom Mag 19(1):5061. https://doi.org/10.1109/MRA.2012.2184671

50. Neumann $P$, Hernandez V, Lilienthal A, Bartholmai M, Schiller J (2013) Gas source localization with a micro-drone using bio-inspired and particle filter-based algorithms. Adv Robot 27(9):725-738. https://doi.org/10.1080/01691864.2013.779052

51. Nisky I, Pressman A, Pugh C, Mussa-Ivaldi F, Karniel A (2011) Perception and action in teleoperated needle insertion. IEEE Trans Haptics 4(3):155-166. https://doi.org/10.1109/TOH.2011.30

52. Phillips N, Ackley R, Crosson E, Down A, Hutyra L, Brondfield M, Karr J, Zhao K, Jackson R (2013) Mapping urban pipeline leaks: methane leaks across boston. Environ Pollut 173:1-4. https:// doi.org/10.1016/j.envpol.2012.11.003

53. Roberts R, Barajas M, Rodriguez-Leal E, Gordillo JL (2017) Haptic feedback and visual servoing of teleoperated unmanned aerial vehicle for obstacle awareness and avoidance. Int J Adv Robot Syst 14(4):1729881417716365. https://doi.org/10.1177/17298 81417716365

54. Roberts R, Rodriguez-Leal E (2015) Kinematic and workspacebased dimensional optimization of a 2-dof mechanism for a novel multipoint device. Mechatronics 28:103-114. https://doi. org/10.1016/j.mechatronics.2015.04.011 
55. Roberts R, Rodriguez-Leal E (2015) Kinematic and workspacebased synthesis of a 2-dof mechanism for haptic applications. In: Ceccarelli M, Hernandez E (eds) Multibody mechatronic systems, mechanisms and machine science, vol 25. Springer International Publishing, Berlin, pp 1-9

56. Roberts R, Rodriguez-Leal E (2016) Kinematics and workspacebased dimensional optimization of a novel haptic device for assisted navigation. Mech Based Des Struct Mach 44(1-2):43-57. https://doi.org/10.1080/15397734.2015.1035784

57. Samur E, Flaction L, Bleuler H (2012) Design and evaluation of a novel haptic interface for endoscopic simulation. IEEE Trans Haptics 5(4):301-311. https://doi.org/10.1109/TOH.2011.70

58. Scheggi S, Aggravi M, Prattichizzo D (2017) Cooperative navigation for mixed human-robot teams using haptic feedback. IEEE Trans Human-Mach Syst 47(4):462-473. https://doi.org/10.1109/ THMS.2016.2608936

59. Tzovaras D, Nikolakis G, Fergadis G, Malasiotis S, Stavrakis M (2004) Design and implementation of haptic virtual environments for the training of the visually impaired. IEEE Trans Neural Syst Rehabil Eng 12(2):266-278. https://doi.org/10.1109/TNSRE .2004 .828756

60. Villarreal BL, Gordillo JL (2016) Bioinspired smell sensor: Nostril model and design. IEEE/ASME Trans Mechatron 21(2):912-921. https://doi.org/10.1109/TMECH.2015.2487260

61. Villarreal BL, Olague G, Gordillo J (2016) Synthesis of odor tracking algorithms with genetic programming. Neurocomputing 175(Part B):1019-1032. https://doi.org/10.1016/j.neuco m.2015.09.108
62. Villarreal BL, Olague G, Gordillo JL (2014) Odor plume tracking algorithm inspired on evolution. In: 6th Mexican Conference on Pattern Recognition, Lecture Notes in Computer Science, vol 8495, pp 321-330. Springer International Publishing: Switzerland

63. Wang X, Tao Y, Tao X, Chen J, Jin Y, Shan Z, Tan J, Cao Q, Pan T (2018) An original design of remote robot-assisted intubation system. Scientific reports, vol 8

64. Yuan H, Xiao C, Zhan W, Wang Y, Shi C, Ye H, Jiang K, Ye Z, Zhou C, Wen Y, Li Q (2018) Target detection, positioning and tracking using new uav gas sensor systems: simulation and analysis. J Intell Robot Syst https://doi.org/10.1007/s10846-018-0909-2

65. Zarzhitsky D, Spears D (2005) Swarm approach to chemical source localization. In: 2005 IEEE international conference on systems, man and cybernetics, vol 2, pp 1435-1440. https://doi. org/10.1109/ICSMC.2005.1571348

66. Zilles C, Salisbury J (1995) A constraint-based god-object method for haptic display. In: Proceedings of 1995 IEEE/RSJ international conference on intelligent robots and systems 95 , human robot interaction and cooperative robots, vol 3, pp 146-151. https://doi.org/10.1109/IROS.1995.525876

Publisher's Note Springer Nature remains neutral with regard to jurisdictional claims in published maps and institutional affiliations. 